\section{0th European Meeting on Glial Cells in Health and Disease}

\section{Arthur M Butt* \& Virginia Bay}

Institute of Biomolecular \& Biomedical Sciences, School of Pharmacy \& Biomedical Science, University of Portsmouth, White Swan Road, Portsmouth PO1 2DT, UK

*Author for correspondence: arthur.butt@port.ac.uk

\section{0th European Meeting on Glial Cells in Health and Disease}

Prague Conference Centre, Prague, Czech Republic, 13-17 September 2011

Glial cells are the most abundant cells in the nervous system and have the overall function of ensuring the integrity of neurons and neuronal signaling. There are many different types of glial cells and they are involved in all pathologies of the nervous system. As such, glia are important targets in novel therapeutic strategies. The 10th European Meeting on Glial Cells in Health and Disease is the largest conference of its kind in the world, specialising in the most recent advances in glial cell biology. This meeting brought together the leading international scientists studying the physiology and pathology of glial cells in a 5-day conference, with eight plenary lectures, 32 symposia with 128 lectures from world-renowned experts, poster sessions with over 480 posters summarizing new experimental data, plus workshops and special lectures on state-of-the-art techniques.

Research into the functioning of the nervous system and the causes of nervous system diseases has most often focused on the neurons. This is hardly surprising, since integration of neuronal signaling is responsible for all of our actions and behaviours. It is the disturbance of neuronal activity that manifests itself in the majority of neurological diseases. However, major advances in glial research show that neurons cannot function without their partners - glial cells. The meeting opened with an introductory course on glial biology, in which H Kettenmann, (Germany), President of the Federation of European Neuroscience Societies, together with other leaders introduced the main types of glia and their functions - astrocytes, oligodendrocytes, microglia, Schwann cells and NG2-glia. The meeting continued over the following 5 days with specialized symposia, which focused on specific aspects of glial cells and their functions in health and disease.

Major advances in the study of glial cells have been achieved because of the development of transgenic mice in which specific glial cells can be identified and visualized in the living animal. In his plenary lecture, "Genetic mouse models and imaging approaches to study neuron-glia interactions in vivo", F Kirchhoff (Germany) showed how we can use cell fate tracking and targeted manipulation of glia-specific genes to determine their precise functions. This theme continued in the plenary lecture by B Richardson (UK) who has shown that NG2-glia are essential for myelin development, plasticity and repair. Another key area is the discovery that microglial cells, which were considered to be quiescent unless the CNS was attacked, are highly dynamic and continuously monitor the neuronal environment. This was shown to allow microglia to respond rapidly, within minutes, to any pathological insults in the CNS. Microglia act as the immune system of the brain and are central to immune-mediated damage via the release of cytokines and other factors, as highlighted in the symposia "Immune-mediated destruction of glial cells: mechanisms and consequences", with particular reference to multiple sclerosis, "Microglial clearance function in health and disease" and "Acquiring a double-edged sword phenotype - phagocytic microglia", which dwelt on microglial phagocytosis in multiple sclerosis and neuronal plasticity, with reference to neurodegenerative diseases such as Alzheimer's disease. The symposia "Intrinsic determinants of microglial cell behaviour and function" and "Microglia in neuroinflammation and neurodegeneration", looked at new factors that have been identified that regulate microglia and are therefore potential therapeutic targets for modulating the immune response. In the symposium "New role of glia in pain signaling”, M Tsuda (Japan) presented his ground-breaking work showing that microglia are intrinsic to neuropathic pain. The number of talks and posters dedicated to microglia highlights the growing recognition of these cells in all CNS diseases.

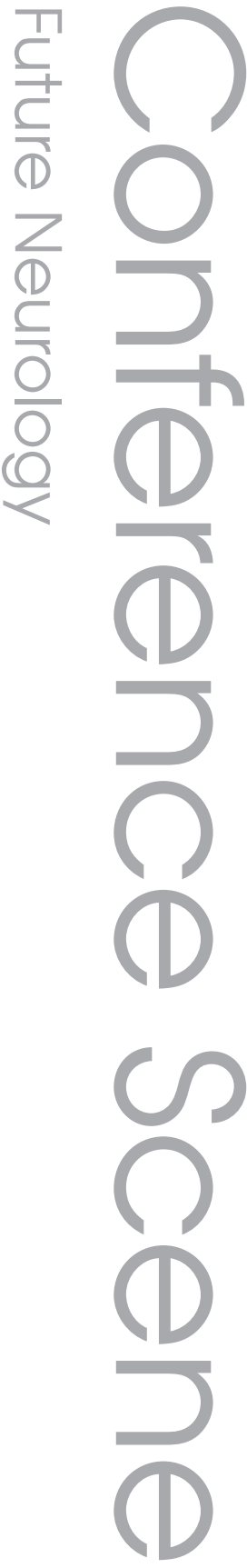

\section{Keywords}

- Alzheimer's disease

- astrocyte = atherolateral

sclerosis $\approx$ epilepsy $\approx$ microglia - multiple sclerosis = neural stem cell $=$ oligodendrocyte

\section{Future Medicine part of}


The main kind of glial cell in the CNS are astrocytes, and one of their main functions is to regulate the neuronal environment. Although these functions have been known for many years, two symposia highlighted new roles that have been identified for specific glutamate transporters, GLT-1/EAAT2, and potassium channels, Kir4.1, in the pathology of atherolateral sclerosis, or motor neuron disease and epilepsy. Glutamate is the main excitatory neurotransmitter in the brain and termination of glutamate activity at neuronal synapses is dependent on glutamate uptake by astrocytes. However, dysregulation of glutamate uptake results in a build-up of glutamate and neuronal excitotoxicity. In the symposium "Regulation of astroglial glutamate transporters in health and disease", E Hermans provided key evidence of the importance of glutamate uptake in astrocytes in the pathology of atherolateral sclerosis, whilst M Rattray (UK) and D Trotti (USA) provided experimental data on how these glutamate transporters are regulated, and J Rothstein (USA) discussed new ways in which they may be therapeutically targeted. During neuronal activity, each action potential results in the release of potassium into the brain extracellular fluid. P Kofuji (USA) and S Skatchkov (Puerto Rico) presented their new evidence that astrocyte Kir4.1 channels are absolutely essential for potassium uptake and their dysfunction results in seizure activity in the symposium "Regulation of glial Kir4.1 channels under physiological and pathophysiological conditions". E Scemes (USA) provided evidence that another astroglial protein, Pannexin-1, is involved in status epilepticus.

These symposia highlight the essential neuron-supporting functions of astrocytes, but astrocytes are also involved in CNS pathology and are major impediments to regeneration. The 'Jeckyll and Hyde' nature of astrocytes was the focus of the symposium "Astrocytes: friend and foe in CNS pathology", which focused on the dual functionality of astrocytes in neuronal signaling (A Butt, UK), multiple sclerosis (S Barnett, UK), spinal injury (F Perin, Spain) and Alzheimer's disease (JJ Rodriguez Arellano, Spain). In the symposium "Reactive astrocytes in neurotrauma and stroke", M Chopp (USA), M Nilsson (Sweden) and T Teng (USA) showed how important astrocytes were in brain remodelling following CNS insults, and that it is not simply a matter of inhibiting astrocytes to promote CNS recovery.
M Götz (Germany), in her plenary lecture, presented a new function for astrocytes - neurogenesis. She showed that glial cells generate neurons throughout life and identified key transduction mechanisms that are responsible for reprogramming astrocytes into neural stem cells. This was the subject of a symposium by $\mathrm{E}$ Hol (The Netherlands) and A Bordey (USA), and JM Garcia Verdugo (Spain) asked the critical question of how neurogenesis differs in the adult mouse and human brain. The answer turns out to be that the mouse and human are very different, which was reiterated by M Nedergaard (USA), who has shown that human astrocytes are far more complex than their rodent counterparts. The study of human material remains essential, and continues to provide new insights, with R Bartesaghi (Italy) and A Eisch (USA) describing their evidence, respectively, that widespread neurogenesis impairment during early brain development is a key determinant of neurological dysfunction in Down's syndrome, and that adult neurogenesis is important in psychiatric disorders.

In summary, glial cells have a multitude of functions that are essential for maintaining CNS functionality, are central to and often initiating factors in all CNS pathologies, and are essential for neurogenesis and repair. Identiftying new means of targeting glial cells was therefore a major emphasis of the conference, with clear headway towards future therapeutic application in multiple sclerosis, Alzheimer's disease, and spinal injury. R Melcangi (Italy) highlighted the need to search for innovative tools for neuroprotection, providing compelling evidence in support of sex-specific neuroprotective therapies based on neuroactive steroids. The work of M Götz on neurogenic astrocytes has neuroscientists very excited, and these cells are clear targets in CNS diseases and injury. The abstracts are published in the journal Glia (Wiley-Blackwell, Hoboken, NJ, USA).

\footnotetext{
Financial \& competing interests disclosure

The authors' research presented at the meeting was funded by the Medical Research Council, UK. The authors have no other relevant affiliations or financial involvement with any organization or entity with a financial interest in or financial conflict with the subject matter or materials discussed in the manuscript apart from those disclosed.

No writing assistance was utilized in the production of this manuscript.
} 